\section{PTU-126 MR ASSESSMENT OF CROHN'S RELATED STRICTURES CORRELATES WELL WITH ENDOSCOPIC FINDINGS FACILITATING SAFE AND EFFECTIVE FLUOROSCOPIC DILATATION}

doi:10.1136/gutjnl-2012-302514c.126

${ }^{1} \mathrm{~N}$ Mohammed, ${ }^{*}{ }^{1} \mathrm{~F}$ Majeed, ${ }^{2} \mathrm{D}$ Tolan, ${ }^{2} \mathrm{R}$ Hyland, ${ }^{1} \mathrm{~J}$ Hamlin. ${ }^{1}$ Gastroenterology, Leeds Teaching Hospitals NHS Trust, Leeds, UK; ${ }^{2}$ Radiology, Leeds Teaching Hospitals NHS Trust, Leeds, UK

Introduction Intestinal strictures are a known complication of Crohn's disease (CD) and may be inflammatory (in part), fibrostenotic or post-operative (anastomotic). Treatment options include a combination of medical, endoscopic or surgical interventions. We performed a retrospective analysis of our radiological assessment and endoscopic management of CD related strictures.

Methods A retrospective review of adult patients who underwent balloon dilatation of $\mathrm{CD}$ related strictures by a single endoscopist at our institution. All patients underwent MR enterography prior to endoscopic assessment. Where necessary strictures were dilated under fluoroscopic screening. Endoscopic success was defined as the ability to traverse the stricture endoscopically after dilatation. Clinical success was defined as improvement in patients symptoms at follow-up. Complications, need for escalation of medical therapy, further dilatation or surgical intervention were recorded.

Results A total of 56 dilatations were performed in 30 patients (range $1-5)$. Mean age was 47.5 years. 16 were females. Mean duration of disease was 209 months (range 14-444). Mean follow-up was 29.5 months (range 1-135). 27/30 (90\%) had at least one previous CD surgical resection (range $0-6$, mean 1.96 per patient). The site of the strictures were ileo-colonic in $21 / 30(70 \%)$, colonic $3 / 30(10 \%)$, gastro-duodenal $3 / 30(10 \%)$, ileo-rectal $2 / 30(7 \%)$ and ileal pouch stricture in 1/30 (3\%). Stricture lengths at MRE were $6 \mathrm{~cm}$, a length deemed significant as this is the length of the colonoscopic balloons. At MRE 17 (57\%) of strictures were deemed to have an inflammatory component and 13 (43\%) fibrostenotic. There was correlation between MRE and endoscopic findings of the nature of the stricturing (inflammatory vs fibrostenotic) in 26/30 (87\%) of cases. Fluoroscopic screening was used in $21 / 30(70 \%)$ of cases. Dilatation endoscopically successful in $27 / 30(90 \%)$ cases and clinically successful in $26 / 30$ (87\%) of cases. No dilatation was performed in one case due to technical difficulties and this patient ultimately required surgical resection. Fourteen patients (47\%) required repeated dilatations for symptom recurrence (range $2-5$ dilatations). 17 patients $(57 \%$ ) had an escalation of their medical therapy after dilatation. A total of $5 / 30$ $(17 \%)$ ultimately required elective surgery for symptom recurrence. Conclusion MRE enterographic assessment of CD related strictures correlates well with endoscopic findings. Fluoroscopic screening facilitates safe and effective dilatation of CD related strictures which, together with optimising medical therapy, can reduce the need for surgical intervention.

Competing interests None declared.

\section{PTU-127 THE IMPACT OF SURGERY ON HEALTH RELATED QUALITY OF LIFE IN ULCERATIVE COLITIS}

doi:10.1136/gutjpl-2012-302514c.127

${ }^{1} \mathrm{P}$ Swinburn, ${ }^{*}{ }^{1} \mathrm{H}$ Elwick, ${ }^{2} \mathrm{~K}$ Bean, ${ }^{2} \mathrm{~A}$ Curry, ${ }^{2} \mathrm{~S}$ Patel, ${ }^{3} \mathrm{~K}$ Bodger, ${ }^{1} \mathrm{~A}$ Lloyd. ${ }^{1}$ Patient Reported Outcomes, Oxford Outcomes, Oxford, UK; ${ }^{2}$ Medical Division, Abbott Laboratories, Maidenhead, UK; ${ }^{3}$ Gastroenterology, University Hospital Aintree, Liverpool, UK

Introduction Surgery is perceived as curative for ulcerative colitis but may not restore full health. Health-related QoL in ulcerative colitis is often measured using disease-specific instruments (eg, the Inflammatory Bowel Disease Questionnaire, IBD-Q) and less commonly using generic instruments (eg, EuroQol EQ5D). The generic approach measures QoL on a common "utility" scale ranging from 0 (dead) to 1 (full health). This allows for more useful comparison of impaired QoL relative to a healthy population or to individuals with other diseases. To examine the impact on surgery on patients' QoL, this study captured data on both disease-specific and generic GoL on patients post-colectomy where published data are limited, as well as patients without colectomy. Utility scores of patients across the full spectrum of disease severity in UC were also evaluated.

Methods 230 UC patients (including 30 post-surgery patients) were recruited along with 100 age and gender matched controls. Participants completed an online survey which comprised the Simple Clinical Colitis Activity Index, EuroOol EQ-5D and the IBD-O. Basic sociodemographic and clinical data were also collected. Disease severity was categorised using established cut-off values for the IBDQ. EQ-5D utility scores were compared across disease severity, among post-surgery patients vs non-surgery patients, and among post-surgery patient vs controls.

Results EQ-5D utility scores demonstrate a clear relationship with disease severity as categorised by the IBDO (ANOVA F $(2,329)=$ $5.544, \mathrm{p}=0.004)$ with post-surgery patients reporting poorer $\mathrm{QoL}$ than non-surgery patients $(p=0.016)$ or controls $(p=0.03)$. Those with the most severe disease report values comparable to colorectal cancer (Tappenden, 2007).

Conclusion Global QoL (utilities) among post-surgical patients was significantly poorer than both age and gender matched population controls and patients in remission or with mildly active colitis, suggesting that identification of effective new drug therapies for severe disease remains the goal for optimising long term $\mathrm{QoL}$.

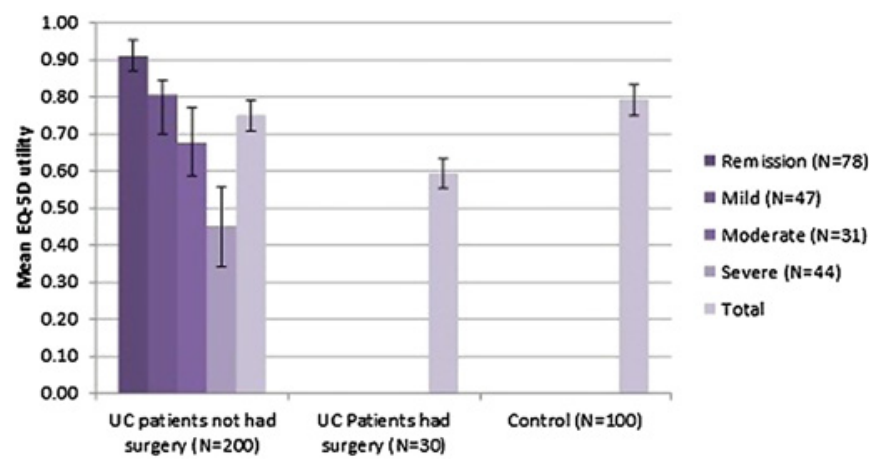

Abstract PTU-127 Figure 1 Mean E0-5D utility scores for study participants $(n=330)$.

Competing interests $\mathrm{P}$ Swinburn Grant/Research Support from: Abbott Laboratories Ltd, H Elwick Grant/Research Support from: Abbott Laboratories Ltd, K Bean: None Declared, A Curry: None declared, S Patel: None declared, K Bodger Consultant for: Abbott Laboratories Ltd, A Lloyd Grant/Research Support from: Abbott Laboratories Ltd.

\section{REFERENCE}

1. Tappenden $\mathbf{P}$, Chilcott J, Eggington S, et al. Option appraisal of population-based colorectal cancer screening programmes in England. Gut 2007:56:677-84.

\section{PTU-128 TEMPORARY DOUBLE-DOSING WITH INFLIXIMAB: QUICK FIX OR LONG TERM SOLUTION?}

doi:10.1136/gutjnl-2012-302514c.128

${ }^{1} \mathrm{P}$ M Irving, * ${ }^{2} S$ Shinhmar, ${ }^{1} J$ Duncan, ${ }^{1} M$ Sastrillo, 'S Anderson, ${ }^{1} J$ Sanderson

2J 0 Lindsay. 'Gastroenterology, Guy's and St Thomas' Hospital, London, UK;

${ }^{2}$ Gastroenterology, Barts and The London, London, UK

Introduction The increasing use of anti-TNF drugs in Crohn's disease reflects their efficacy. Unfortunately, even with scheduled 
maintenance therapy, secondary loss of response is common affecting approximately $10 \%-15 \%$ of patients/year. Increasing drug levels, whether by doubling the dose or decreasing the interval, recaptures response in approximately $75 \%$ of patients. However, both of these strategies have cost implications. Anecdotal reports suggest that in patients losing response on maintenance infliximab (IFX) $5 \mathrm{mg} / \mathrm{kg}$, a temporary increase to double doses (DD; $10 \mathrm{mg} / \mathrm{kg}$ ) can lead to subsequent recapture of response at the lower dose. In a small cohort, we have previously shown that this strategy was not viable in the majority of patients. We now present an extended cohort across two centres with longer follow-up.

Methods We performed a retrospective review across two tertiary centres of all patients with Crohn's disease who had received temporary increases to DD of IFX for loss of response. Demographic data, HBI prior to the first infusion at the higher dose and prior to the first infusion at the lower dose, and ability to continue on IFX at $5 \mathrm{mg} / \mathrm{kg}$ were recorded.

Results 34 patients (18M:16F, median age 24 (range 12-51)) received DD IFX for loss of response. Median disease duration was 3 years (range $0-32$ ) and the median time to dose increase from starting IFX was 12 months (range 3-60). All had received standard induction doses of IFX at $5 \mathrm{mg} / \mathrm{kg}$ on weeks 0,2 and 6 and were on scheduled maintenance therapy. The dose interval prior to dose increase was 8 weeks for 24 patients, 7 weeks for 1 , and 6 weeks for 9. 26 patients were on concurrent immunomodulators and 8 were not. One patient received $4 \mathrm{DD}, 27$ received $2 \mathrm{DD}$ and six patients 1 DD. Dose increase was effective in the short term with the median HBI falling from 6 (range $0-27$ ) prior to the first infusion at $10 \mathrm{mg} /$ $\mathrm{kg}$, to $1(0-7)$ prior to the first infusion back at the standard dose $(5 \mathrm{mg} / \mathrm{kg})(\mathrm{p}=0.003)$. However, only seven patients remained on IFX at the end of follow-up (median 13 months (range 8-25)). The median interval to discontinuing IFX was 4 months after the first DD (1-19). Of those who discontinued, four had infusion reactions while the others failed to maintain a response to IFX.

Conclusion Because of the limited treatment options available in Crohn's disease, attempting to recapture response in patients on IFX is appropriate. However, while temporary double-dosing is effective in the short term, it does not deliver long term disease control after subsequent dose reduction. Whether dose reduction is possible after prolonged dose increase remains to be answered.

Competing interests None declared.

\section{PTU-129 INITIAL EXPERIENCE WITH INFLIXIMAB LEVELS IN A TERTIARY IBD CENTRE}

doi:10.1136/gutjnl-2012-302514c.129

${ }^{1} \mathrm{P} M$ Irving, * ${ }^{2} \mathrm{Z}$ Arkir, ${ }^{1} \mathrm{~J}$ Duncan, ${ }^{1} \mathrm{M}$ Sastrillo, ${ }^{1} \mathrm{~S}$ Anderson, ${ }^{1} \mathrm{~J}$ Sanderson.

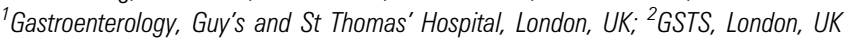

Introduction Biologic use in the UK is increasing in patients with Crohn's disease (CD). While highly effective at inducing and maintaining remission, secondary loss of response occurs in approximately $15 \%$ of patients on maintenance treatment with biologics every year. There is some preliminary evidence that drug levels may play a role in this. However, measurement of anti-TNF levels has not been routinely available in the UK until very recently. Methods We performed a service evaluation of a CE marked kit measuring serum levels of infliximab (IFX) as well as anti-drug antibodies (BMD, Marne La Vallee, France). Samples were taken immediately prior to infusion of IFX in patients attending for infusions over a 9-week period (March-May 2011). Results were not used in clinical management. A retrospective notes review was undertaken in January 2012 to see how drug levels related to clinical activity and to outcome. Only patients with CD on stable maintenance therapy were included and if repeated samples were taken, only the first measurement was used.
Results 52 samples were taken on 41 patients. Eight were excluded either because they did not have CD or because they were still in the induction phase of treatment. 33 patients ( 17 male) were, therefore, included in the analysis. Seven had subtherapeutic levels (SL) of IFX $(<2 \mu \mathrm{g} / \mathrm{ml})$. Patients with SL had a higher median Harvey Bradshaw index (HBI) (5 (range $0-7)$ ) and $\mathrm{C}$ reactive protein (CRP) $(8(0-50))$ at the time the sample was taken than those with therapeutic levels (TL) (HBI $(1(0-8) p=0.03$ : CRP $0(0-8) p<0.05)$. The highest HBI and CRP recorded during subsequent follow-up was also higher in those with SL than TL but not significantly so (HBI SL 4 (2-11) vs TL $2(0-12)$ : CRP SL $11(0-56)$ vs TL $0(0-21))$. Patients with SL were also more likely to require dose escalation of IFX (SL 2/7: TL 0/ $26(p<0.04))$ and intervention (change of drug therapy or surgical/ endoscopic intervention) (SL 3/7: TL 2/27 ( $p=0.05)$ ).

Conclusion Subtherapeutic IFX levels were associated with increased disease activity defined by biomarkers (CRP) and disease activity scores (HBI). Subtherapeutic levels were also predictive of a worse disease course over the following 6-8 months. Measuring anti-TNF drug levels in patients with IBD is promising and the utility of these tests in every day practice should be investigated further.

Competing interests None declared.

\section{PTU-130 MUCOSAL MRNA EXPRESSION PROFILING FROM THE TERMINAL ILEUM AND COLON REVEALS UNDER EXPRESSION OF CLAUDIN 8, A TIGHT JUNCTION MOLECULE, AS POTENTIALLY CAUSAL IN ULCERATIVE COLITIS}

doi:10.1136/gutjnl-2012-302514c.130

${ }^{1} \mathrm{P}$ J Smith, ${ }^{*}{ }^{1} \mathrm{~A}$ P Levine, ${ }^{1} \mathrm{G} W$ Sewell, ${ }^{1} \mathrm{~N}$ R O'Shea, ${ }^{2} \mathrm{R}$ Vega, ${ }^{2} \mathrm{~S}$ L Bloom, ${ }^{1} \mathrm{~A}$ M Smith, ${ }^{1} \mathrm{~A}$ W Segal. ${ }^{1}$ Department of Medicine, University College London, London, UK; ${ }^{2}$ Department of Gastroenterology, University College London Hospital, London, UK

Introduction Intestinal barrier dysfunction plays an important role in the pathogenesis of ulcerative colitis (UC). We investigated mRNA profiles of mucosa from the colon and terminal ileum, in patients with UC and controls (HC) to identify genes that might be implicated in the pathogenesis of the disease.

Methods Mucosal biopsies were taken from 24 quiescent UC patients (Mayo score $<3$ ) and $33 \mathrm{HCs}$ undergoing colonoscopy. Patients were on no treatment or on 5-aminosalicylates \pm azathioprine. HCs were patients without organic disease. Parallel biopsies were taken for RNA extraction and histology from macroscopically non-inflamed mucosa in the terminal ileum (TI), ascending, descending and sigmoid colon, and rectum. cRNA was hybridised to Illumina HumanHT v12.0 Expression Beadchips. Expression data were log transformed and normalised. Probes with a detection $p$ value $<0.01$ were analysed. Comparing 85 biopsies from HCs and 68 biopsies from UC patients across the colon, the data for each bowel location were adjusted to the mean $\mathrm{HC}$ rectal expression level. Where multiple biopsies were taken from the same individual, the adjusted data across all biopsies for that individual were averaged. $T$ tests between groups and outlier analysis $(p<0.005$, fold change $(\mathrm{FC}) \geq 1.5$ ) were performed using proprietary software.

Results Of the $\sim 30 \mathrm{~K}$ probes analysed, the two most significantly under-expressed in UC in the colon were those of claudin 8 (CLDN8) with FCs $2.94\left(p=1.29 \times 10^{-5}\right)$ and $3.45\left(p=3.92 \times 10^{-5}\right)$. The expression of claudin 8 increased distally in the colon, whereas claudins 3, 7 and 23 were highly, and uniformly, expressed throughout the colon and were normal in UC. Outlier analysis between HC and UC showed CLDN8 to be significantly underexpressed in $25 \%>40 \%$ of UC patients at all 4 colonic sites. There were no CLDN8 UC outliers in the TI. Most of these outlier patients demonstrated consistent levels of under-expression 